\title{
Effect of Neoadjuvant Chemoradiotherapy on Pathologic Stage and Survival in Patients with Locally Advanced Esophageal Cancer
}

\author{
Karapetyan $\mathrm{L}^{1}$, Burtness $\mathrm{B}^{2}$, Forastiere $\mathrm{A}^{3}$, Heitmiller $\mathrm{RF}^{4}$, Heath $\mathrm{E}^{5}$ and Gibson $\mathrm{MK}^{* 1}$ \\ ${ }^{1}$ University Hospitals Case Medical Center, Case Western Reserve University, Department of Hematology/Oncology, \\ Cleveland, Ohio, United States \\ ${ }^{2}$ Yale Cancer Center, Yale University School of Medicine, Department of Oncology, New Haven, Connecticut, United \\ States \\ ${ }^{3}$ The Sidney Kimmel Comprehensive Cancer Center at Johns Hopkins, Johns Hopkins University, Division of \\ Medical Oncology, Baltimore, United States \\ ${ }^{4}$ Medstar Union Memorial Hospital, Department of General Surgery, Baltimore, United States
}

${ }^{5}$ Karmanos Cancer Institute, Wayne State University, Department of Medical Oncology, Detroit, Michigan, United States

*Corresponding author: Gibson MK, University Hospitals Case Medical Center, Case Western Reserve University, Department of Hematology/Oncology, Cleveland, Ohio, United States, Fax: 216-844-5234, Tel: 216-844-0513, E-mail: Michael.gibson@uhhospitals.org

Citation: Karapetyan L, Burtness B, Forastiere A, Heitmiller RF, Heath E, et al. (2016) Effect of Neoadjuvant Chemoradiotherapy on Pathologic Stage and Survival in Patients with Locally Advanced Esophageal Cancer. J Cancer Sci Clin Oncol 3(1): 105. doi: 10.15744/2394-6520.3.105

Received Date: March 16, 2016 Accepted Date: June 06, 2016 Published Date: June 09, 2016

\begin{abstract}
Background: The treatment of resectable stages of esophageal cancer often involves pre-operative concurrent chemoradiotherapy. This study aims to evaluate the effect of cisplatin/5-fluorouracil (5-FU)-based neoadjuvant chemoradiotherapy on pathologic stage and survival in patients with locally advanced esophageal cancer.
\end{abstract}

Methods: Patients $(\mathrm{n}=42)$ received cisplatin/5-FU-based induction chemoradiotherapy followed by esophagectomy. Survival calculated from time of surgery was stratified by pathologic stage and controlled for clinical factors.

Results: Clinical factors included: age (range 35 to 75 years); sex (38 male; 4 female); and histology (31 adeno; 11 squamous cell). The pre-treatment stage distribution included: stage IIA $(n=9)$, stage IIB $(n=3)$, stage III $(n=21)$ and stage IV $(n=9)$. Three patients did not undergo surgery (2 progressive disease; 1 refused) and in one patient pathologic stage was not determined. Pre-operative chemoradiotherapy downstaged 29 patients and resulted in no change in 6 patients. Another 6 patients progressed. The post-treatment stage distribution included: stage $0(n=12)$, stage I $(n=2)$, stage IIA $(n=13)$, stage III $(n=3)$ and stage IV $(n=6)$. The median survival is 4 years with minimum follow up of surviving patients of 5.5 years. Median survivals for each pathologic stage were: stage 0 ( 7 yrs), stage I (not reached), stage IIA (1.9 yrs), stage IIB (1 yr), stage III (2.2 yrs) and stage IV (0.8 yr). Higher pathological stage (adjusted for clinical co-variates) correlated with worse survival (HR 1.56; 95\% CI 1.16-2.1).

Conclusion: Cisplatin/5-FU-based neoadjuvant chemoradiotherapy for resectable esophageal cancer leads to pathologic downstaging in most patients, and lower pathologic stage correlates with better survival.

Keywords: Esophageal cancer; Chemoradiotherapy; Pathological staging; Overall survival

\section{Introduction}

Although esophageal cancer comprises a small percentage (1.5\%) of total cancer cases in the United States, mortality rate remains high. In the United States, an estimated 16,980 cases of esophageal cancer will be diagnosed in 2015 and 15,590 deaths are expected from the disease $[1,2]$. Regardless of histology, approximately $50-60 \%$ of patients present with incurable locally advance or metastatic disease. As such, at most 10-15\% of all patients presenting with esophageal cancer are cured [3]. For those with locally advanced, non-metastatic disease, cure is achieved in up to $40 \%$ of patients when multimodality therapy is used $[4,5]$.

A significant though modest improvement in the relative 5-year survival rate is reported from the SEER database for patients diagnosed between 1974 and 2007 (5\% for the period 1974-1976, 8\% for 1983-1985, 14\% for 1995-2000, and 18\% for 2001-2007); however, survival rates remain low [6].

Of the subset of patients with locally advanced, and thus potentially curable disease, stages II-IV are most often found. These stages involve tumor invasion at least into the muscularis propria (T2) and/or involvement of regional nodes (N1) or spread to 
celiac or cervical nodes (M1a) from a distal or upper thoracic primary, respectively. Sub-clinical micro-metastatic disease is also present in a majority of patients. This multitude of factors explains why, while surgery remains the mainstay of treatment for operable disease, local and distant recurrences after surgery alone are common.

To improve on the outcomes achieved with surgery alone, the addition of chemotherapy (CT) and radiotherapy (RT) to surgery has been tested in various combinations and sequences [5,7-11]. Treatment with CT early may reduce both loco-regional and micro-metastatic tumor burden, while RT may downstage tumor, sterilizing areas that will not be affected by surgery. When administered together in a concurrent fashion, chemoradiotherapy (CRT) exploits the ability of cytotoxics such as cisplatin and 5-fluorouracil (5-FU) to enhance RT effects and prevent emergence of drug resistant clones. Administering these therapies before surgery takes advantage of enhanced drug delivery to tumor while the vasculature is intact [12].

A reproducible result of concomitant CRT is the achievement of a pathologic complete response (pCR) in the resected esophageal specimen in a proportion of patients. Although at most $25-30 \%$ of patients attain a pCR, those that do have a much higher survival rate than those patients who have residual disease. The finding of pCR as a correlative marker for improved survival following CRT is demonstrated across multiple studies; however, as a binary outcome marker (pCR vs not), it does not provide prognostic information for the $70-75 \%$ of patients who do not achieve a pCR. The goal of this study is to evaluate pathologic stage as a categorical variable for predicting survival in patients with locally advanced esophageal cancer treated with pre-operative CRT and to determine whether pathologic stage better predicted outcome than pretreatment clinical stage.

\section{Material and Methods}

\section{Eligibility criteria}

From March 1995 to November 1997, a total of 42 patients with histologically confirmed invasive squamous cell carcinoma or adenocarcinoma of the esophagus or gastroesophageal junction were enrolled on a phase II clinical trial at the Johns Hopkins Cancer Center, Baltimore, MD, and the Yale University Cancer Center, New Haven, CT. Because trial was conducted between 19951997, it was not registered in ClinicalTrials.gov. All patients provided informed consent per institutional review board guidelines. All patients were newly diagnosed and had received no prior treatment. Each patient was older than 18 years and had a Karnofsky performance status greater than $60 \%$ (if younger than 70 years) or greater than $80 \%$ (if 70 years or older) with adequate hepatic, renal and bone marrow reserve. The disease was limited to the primary and regional nodes, although celiac nodal involvement (M1a) was permitted for primary tumors in the mid, distal or gastroesophageal junction. Patients were required to be surgical candidates with disease that could be encompassed in a single radiation port. All patients were staged with history taking, physical examination, routine hematologic and biochemical tests, computed tomography (CT) scans of the chest, abdomen and pelvis, endoscopic ultrasound (EUS) and exploratory laparoscopy.

\section{Treatment Plan}

Preoperative chemotherapy and radiotherapy were administered over 30 calendar days. Cisplatin at a dose of $20 \mathrm{mg} / \mathrm{m}^{2} / \mathrm{day}$ was administered on days 1-5 and 26-30 and 5-FU at a dose of $225 \mathrm{mg} / \mathrm{m}^{2} /$ day was given on days $1-30$ by continuous IV infusion. Radiotherapy was given at a daily dose of 2 Gy to a total dose of $44 \mathrm{~Gy}$. Esophagogastrectomy was carried out approximately 4 weeks after completion of chemoradiotherapy in those patients without disease progression. Partial esophagogastrectomy with one-field lymphadenectomy was performed in this study. Patients were subsequently treated with adjuvant chemotherapy consisting of paclitaxel $135 \mathrm{mg} / \mathrm{m}^{2}$ IV for 24 hours on day 1 and cisplatin $75 \mathrm{mg} / \mathrm{m}^{2} \mathrm{IV}$ on day 2 repeated every 3 weeks for three cycles. Adjuvant chemotherapy was planned for all patients who had negative margins (R0 resection).

The NIH CTC criteria (http://ctep.info.nih.gov/reporting/ctc.html) were used to measure toxicity, and appropriate dose adjustments and delays were made. All patients provided written informed consent, and the protocol was approved by the institutional review boards at Johns Hopkins University and Yale University.

Follow-up after treatment involved medical oncology visits at four-month intervals for the first year and at six-month intervals for the second through fifth years. After five years, patients were evaluated annually.

The primary outcome was pathologic response in the resected specimen. A pathologic complete response (pCR) was defined as the histological absence of residual tumor in the resected esophageal specimen and nodal tissue. A partial response (PR) was defined as residual malignant cells in the resected specimen. Progressive disease (PD) was defined as presence of metastatic or unresectable disease prior to surgery. The secondary outcome, overall survival, was defined as length of the time from surgery until death from any cause or last follow-up. Survival curves were estimated using the Kaplan-Meier method. Pathologic staging followed the standard TNM criteria and was provided on the clinical report by the pathologist initially handling the surgical specimen.

\section{Data and Statistical Analysis}

Follow-up data regarding survival were extracted from oncology medical records maintained at Johns Hopkins Cancer Center and Yale Cancer Center. All living patients had at least 5 years of follow-up. Clinical co-variates were also extracted from the medical 
record and coded as follows: age at enrollment (continuous and binary; $<=65 />65$ ), gender (M/F), Barrett's metaplasia (present/ absent), tumor location (mid/distal), histology (squamous cell carcinoma/ adenocarcinoma), differentiation (categorical; well, moderate, poor). Pathologic stage (obtained from the clinical pathology report) was coded as a categorical variable per the standard TNM/AJCC criteria [0 (pCR); I; IIA; IIB; III; IVa; IVb (progressive disease)].

Survival was determined by the method of Kaplan and Meier. Survival curves were compared using the log-rank test. Univariate and multivariate Cox proportional hazard models were used to investigate the role of clinical and histologic co-variates. Hazard ratios are expressed as mean with $95 \%$ confidence intervals. To investigate for interactions and co-variations, multivariate models were used to adjust for clinical covariates.

All statistical analyses were done using Intercooled Stata ${ }^{\mathrm{TM}}$ version 7 (Stata Corporation, College Station, TX).

\section{Results}

\section{Patient Characteristics and Reponse}

A total of 42 patients were enrolled prospectively on this single arm, phase II clinical trial. Characteristics of all evaluated patients are listed in Table 1. Consistent with the recent epidemiology of esophageal cancer in the United States, the majority of patients had adenocarcinoma (31), were male (38) and harbored disease in the distal esophagus and gastroesophageal junction (36). Pretreatment stage ranged broadly from IIA to IVA. Eleven patients had stage IIA disease, five had stage IIB, 21 had stage III disease, and five had stage IV disease. Barrett's Esophagus was present in 14 of these patients. Of the 42 patients, 39 underwent laparoscopy, and six did not receive an optimal EUS because of obstructing esophageal lesions.

\begin{tabular}{|c|c|c|}
\hline Age at enrollment & Mean (SD) & $61(9)$ \\
\hline \multirow{2}{*}{ Age Range } & Minimum & 35 \\
\hline & Maximum & 75 \\
\hline \multirow{2}{*}{ Gender } & Male & 38 \\
\hline & Female & 4 \\
\hline \multirow{3}{*}{$\begin{array}{l}\text { Pathologic } \\
\text { Response }\end{array}$} & Progressive Disease & 6 \\
\hline & Partial Response & 23 \\
\hline & Complete Response & 12 \\
\hline \multirow{4}{*}{ Location } & Upper & 2 \\
\hline & Middle & 4 \\
\hline & Distal & 31 \\
\hline & GE Junction & 5 \\
\hline \multirow{2}{*}{ Survival } & Alive & 10 \\
\hline & Dead & 30 \\
\hline \multirow{2}{*}{ Barrett's Histology } & Present & 14 \\
\hline & Absent & 28 \\
\hline \multirow{2}{*}{ Histology } & Adenocarcinoma & 31 \\
\hline & Squamous Cell & 11 \\
\hline
\end{tabular}

All 42 patients completed CRT prior to surgery. Twenty-five patients (60\%) received $100 \%$ of the planned total dose of 5-FU (9,000 $\left.\mathrm{mg} / \mathrm{m}^{2}\right)$, and 37 patients $(88 \%)$ received $100 \%$ of the planned total dose of cisplatin $\left(200 \mathrm{mg} / \mathrm{m}^{2}\right)$. Twenty-three patients $(55 \%)$ received $100 \%$ of the doses of both drugs. All 42 patients received $100 \%$ of the total dose of radiation (44 Gy). Three patients did not have surgery. One patient refused surgery, and two patients had progressive disease intra-operatively. Of the 39 patients who underwent surgery, thirty-five had complete tumor resection with negative margins (R0). There was one postoperative death from respiratory failure and aspiration pneumonia 101 days after surgery. 62\% (26/42) of all patients received at least one course of adjuvant therapy with paclitaxel and cisplatin. Preoperative therapy had a significant impact on stage (Table 2). Twenty-nine patients were downstaged, six patients had no change in stage, and six had PD. All patients with unchanged or progressive disease died. Pathological data were available for 41 patients because one patient refused surgery. Twelve patients had pCR, 23 had partial response (PR), and six had PD. The median survival time was 4 years with minimum follow up of surviving patients of 5.5 years. The 5-year survival rate was 33\% (95\% CI [Confidence Interval], 19.3-46.7\%). Pathological stages III and IV (adjusted for clinical 
co-variates similar to age, performance status, clinical T category) correlated with worse survival (HR 1.56; 95\% CI 1.16-2.1). The distribution of survivors by pathologic stage was: stage $0(n=5)$, stage I $(n=2)$, stage IIA $(n=2)$, stage IIB $(n=1)$, stage III $(n=0)$ and stage IV $(n=0)$. The Kaplan-Meier representation of overall survival is presented in Figure 1, and is consistent with historical data [13] (Table 3).

\begin{tabular}{|c|c|c|}
\hline AJCC Stage & $\begin{array}{c}\text { Pre-Treatment } \\
\text { Clinical }\end{array}$ & $\begin{array}{c}\text { Pre-Treatment } \\
\text { Clinical }\end{array}$ \\
\hline 0 & 0 & 0 \\
\hline I & 0 & 0 \\
\hline IIA & 9 & 9 \\
\hline IIB & 3 & 3 \\
\hline III & 21 & 21 \\
\hline IVa & 9 & 9 \\
\hline IVb & 0 & 0 \\
\hline
\end{tabular}

$\mathrm{CR}$, complete response; $\mathrm{NC}$, no change; $\mathrm{PD}$, progressive disease

Table 2: Distribution of Pretreatment (Clinical) and Posttreatment (Pathologic) Stages

\begin{tabular}{|c|c|c|c|}
\hline \multirow{2}{*}{$\begin{array}{c}\text { Effect of } \\
\text { Treatment }\end{array}$} & \multirow{2}{*}{$\begin{array}{c}\text { Effect of } \\
\text { Treatment }\end{array}$} & \multicolumn{2}{|c|}{ Survival Status } \\
\cline { 3 - 4 } & & Alive & Dead \\
\hline Downstaged & Downstaged & 10 & 17 \\
\hline No Change & No Change & 0 & 6 \\
\hline Progressed & Progressed & 0 & 6 \\
\hline
\end{tabular}

Table 3: Impact of Preoperative Chemoradiotherapy on Stage and Relationship to Survival

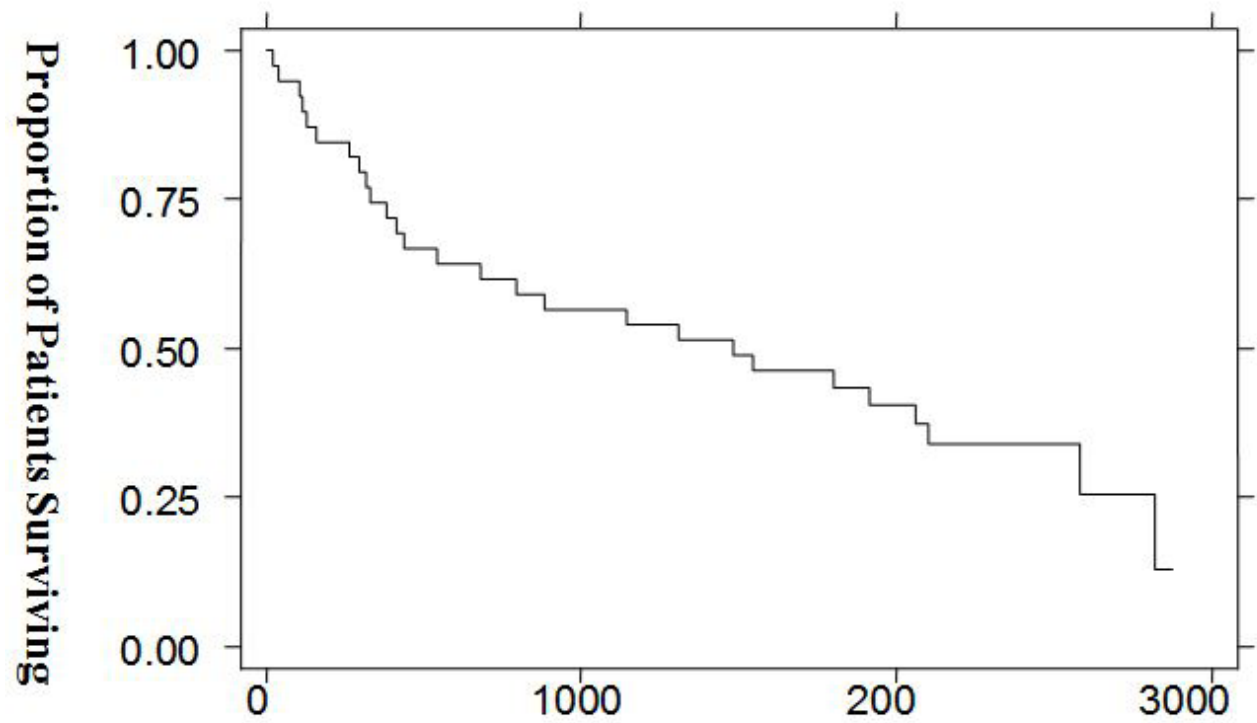

\section{Days from Surgery}

Figure 1: Kaplan-Meier survival analysis of overall survival plotted as the probability of surviving versus time from surgery

\section{Correlation of Clinical and Pathologic Staging Survival}

We next aimed to determine survival stratified by both clinical (pre-operative) and pathologic stage. Figure 2, survival by clinical stage, shows that there is no difference in overall survival when this outcome is stratified by pre-operative stage ( $\mathrm{p}=\mathrm{NS}$ ). In contrast, when OS is stratified by pathologic stage (Figure 3), there is a statistically significant difference $(p=0.05)$. As stage increases, survival decreases for the 39 patients who went to surgery. Further validating this data set and these findings are the results of survival stratified by $\mathrm{pCR}$, which is similar results found by both our group and other investigators $($ Table $4, \mathrm{p}=0.05)$ [13]. 
Kaplan-Meier survival estimates, by preAJCC

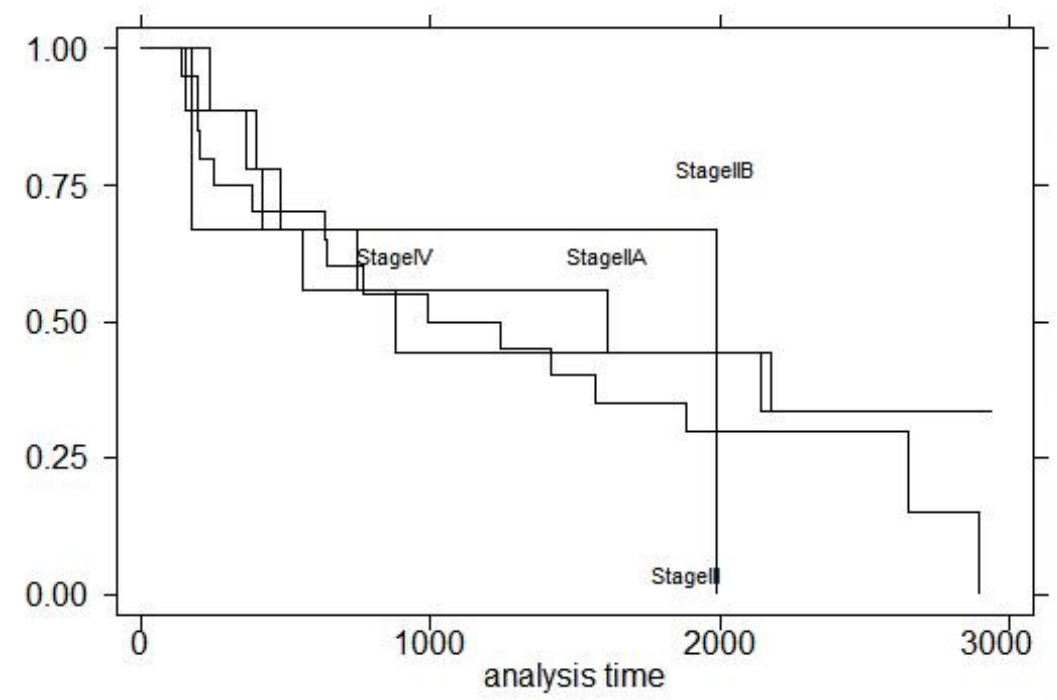

Figure 2: Survival by Clinical (Pre-operative) Stage

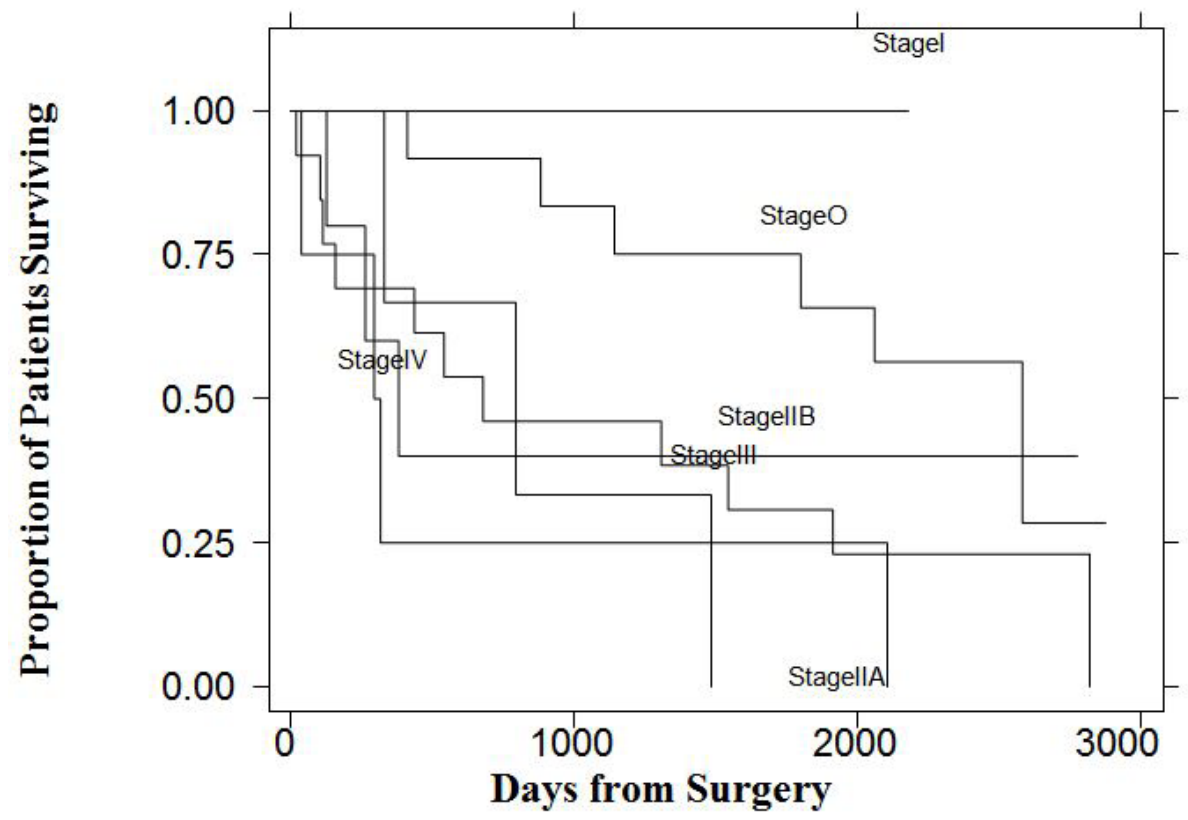

Figure 3: Survival by pathological Stage

\begin{tabular}{|c|c|c|c|c|c|}
\hline \multirow{2}{*}{$\begin{array}{l}\text { Pathologic } \\
\text { Stage }\end{array}$} & \multirow{2}{*}{ Number } & \multicolumn{2}{|c|}{ Survival Status } & \multirow{2}{*}{$\begin{array}{c}\text { Median Survival } \\
\text { (Present study) } \\
\quad n=41\end{array}$} & \multirow{2}{*}{$\begin{array}{c}\text { Median Survival } \\
\text { (historical } \\
\text { control) } n=480\end{array}$} \\
\hline & & alive & dead & & \\
\hline 0 & 12 & 5 & 6 & 7 yrs & Not reached \\
\hline I & 2 & 2 & 0 & Not reached & Not reached \\
\hline IIA & 13 & 2 & 11 & $1.9 \mathrm{yrs}$ & $2.4 \mathrm{yrs}$ \\
\hline IIB & 5 & 1 & 3 & $1 \mathrm{yr}$ & $2.1 \mathrm{yrs}$ \\
\hline III & 3 & 0 & 3 & $2.2 \mathrm{yrs}$ & $1.2 \mathrm{yrs}$ \\
\hline IVa & 2 & 0 & 2 & $0.8 \mathrm{yrs}$ & $0.9 \mathrm{yrs}$ \\
\hline $\mathrm{IVb}$ & 4 & 0 & 4 & $0.8 \mathrm{yrs}$ & $0.9 \mathrm{yrs}$ \\
\hline
\end{tabular}

Table 4: Overall survival by pathological stage vs. historic controls (13) 


\section{Discussion}

Esophageal cancer is a highly lethal malignancy with a poor prognosis [1]. It continues to remain a difficult cancer to treat and a more difficult cancer to cure. The majority of approaches to cure of both adenocarcinoma and squamous cell carcinoma involve surgery, and 5-year overall survival (OS) rate of $15 \%$ to $22 \%$ with surgery alone. Given the poor long term outcome with surgery alone, various approaches are available with the goal of improving control of both loco-regional and systemic micrometastatic disease.

Patients with squamous cell carcinoma can be cured with a primary CRT [14]. By contrast, adenocarcinoma, the most prevalent type with the highest incidence in the United States, requires surgical resection as part of any curative attempt. Multimodality treatment regimen with inclusion of systemic chemotherapy control to prevent the development of distant micrometastatic disease and enhance local radiation effects. One validation of this approach was provided by the results of the intergroup study carried out by Tepper and colleagues [11]. In this study, which failed to accrue to goals, a total of 57 patients were randomized to either pre-operative CRT with cisplatin and 5-FU or surgery alone. The patients treated with the tri-modality approach had a statistically significant improvement in survival. Furthermore, this study again showed that pCR to neoadjuvant CRT is associated with significantly improved survival. In the CROSS trial 368 patients were randomized to either pre-operative CRT with carboplatin and paclitaxel or surgery alone. The median overall survival for patients with SCC of esophagus was 81.6 months (95\% CI 47.2116.0) months in the chemoradiotherapy plus surgery group vs 21.1 months (95\% CI 15.4-26.7) in the surgery group (hazard ratio, $0.48 ; 95 \%$ confidence interval, 0.28 to $0.83 ; \mathrm{P}=0.008$ ). Median overall survival in patients with adenocarcnioma was $43 \cdot 2$ months (24.9-61.4) in the neoadjuvant chemoradiotherapy plus surgery group and $27 \cdot 1$ months $(13 \cdot 0-41 \cdot 2)$ in the surgery alone group (HR 0.73 [95\% CI 0.55-0.98]; log-rank p=0.038) [15].

The work presented here aimed to further refine the relationship between pathologic stage and survival, with the added approach of correlating each pathologic stage with outcome. As shown in Figure 3, there was a statistically significant relationship between post-surgical stage and overall survival. This data suggest that pathologic stage, and not clinical stage, is the best predictor of survival for patients treated with neoadjuvant CRT. This correlation suggests that pre-operative CRT may actually impact the behavior of the disease. In a study by Davies and colleagues, patients who benefitted from a downstaging effect from CT, the 5-year survival was significantly improved compared with patients without response $(52.5 \%$ vs $12.6 \%$, respectively, $\mathrm{p}<0.001)$ [16]. In other words, survival outcome data should be based on pathologic stage, as is the case with primary surgery, with the difference being that the addition of pre-operative CRT may impact the pre-operative prognosis by changing the stage for any individual patient.

This study has several limitations. Because the trial was conducted before 2009, AJCC Cancer Staging $5^{\text {th }}$ edition was used to enroll patients and to classify post-CRT pathologic staging. This is different from AJCC Cancer Staging $7^{\text {th }}$ edition, which is currently used in staging EC. Due to the years of the study, positron emission tomography (PET) was not routinely included as part of staging. PET scan may result in stage migration in up to $10-15 \%$ of patients by detecting previously undescribed metastatic disease. The small sample size of the study may limit the results. Finally, we were not able to gather enough data about patients who received adjuvant therapy, which might impact on patient survival.

This information has several implications for clinical practice. First, prognostic information provided to patients in the pretreatment setting may not reflect the actual prognosis after neoadjuvant CRT followed by surgery. Furthermore, this information may explain why pCR is such a strong predictor of survival. Second, pathologic stage may be the best parameter for determining which patients require adjuvant therapy. Third, this data provides further motivation for the search for predictors of who will most likely respond to pre-operative CRT.

\section{Conclusions}

In summary, this study indicates that most patients are downstaged by induction CRT. All patients with unchanged or progressive disease during CMT died. Survival correlates with pathologic stage not pre-treatment (clinical) stage, and survival by pathologic stage of patients treated with pre-operative CMT compares favorably with similarly staged patients treated with surgery alone. These findings support the use of pre-operative CRT and have implications for the selection of patients for adjuvant treatment as well as the search for determinates of response to induction CRT. Several cooperative group studies are planned to address this issue.

\section{References}

1. Siegel R, Ma J, Zou Z, Jemal A (2014) Cancer statistics, 2014. CA Cancer J Clin 64: 9-29.

2. Arnold M, Soerjomataram I, Ferlay J, Forman D (2014) Global incidence of oesophageal cancer by histological subtype in 2012. Gut 64: 381-7.

3. Pennathur A, Gibson MK, Jobe BA, Luketich JD (2013) Oesophageal carcinoma. Lancet 381: 400-12.

4. van Hagen P, Hulshof MC, van Lanschot JJ, Steyerberg EW, van Berge Henegouwen MI, et al. (2012) Preoperative chemoradiotherapy for esophageal or junctional cancer. N Engl J Med 366: 2074-84. 
5. Bang YJ, Van Cutsem E, Feyereislova A, Chung HC, Shen L, et al. (2010) Trastuzumab in combination with chemotherapy versus chemotherapy alone for treatment of HER2-positive advanced gastric or gastro-oesophageal junction cancer (ToGA): a phase 3, open-label, randomised controlled trial. Lancet 376: 68797.

6. Njei B, McCarty TR, Birk JW (2016) Trends in esophageal cancer survival in United States adults from 1973 to 2009: a SEER database analysis. J Gastroenterol Hepatol doi: 10.1111/jgh.13289.

7. Allum W, Cunningham D, Weeden S (2003) Perioperative chemotherapy in operable gastric and lower oesophageal cancer: Final results of a randomized, controlled trial (the MAGIC trial, ISRCTN 93793971). J Clini Oncol 308 S.

8. Heath EI, Burtness BA, Heitmiller RF, Salem R, Kleinberg L, et al. (2000) Phase II evaluation of preoperative chemoradiation and postoperative adjuvant chemotherapy for squamous cell and adenocarcinoma of the esophagus. J Clin Oncol 18: 868-76.

9. Heath EI, Burtness BA, Kleinberg L, Salem RR, Yang SC, et al. (2006) Phase II, parallel-design study of preoperative combined modality therapy and the matrix metalloprotease (mmp) inhibitor prinomastat in patients with esophageal adenocarcinoma. Invest New Drugs 24: 135-40.

10. Herskovic A, Martz K, al-Sarraf M, Leichman L, Brindle J, et al. (1992) Combined chemotherapy and radiotherapy compared with radiotherapy alone in patients with cancer of the esophagus. N Engl J Med 326: 1593-8.

11. Tepper J, Krasna MJ, Niedzwiecki D, Hollis D, Reed CE, et al. (2008) Phase III trial of trimodality therapy with cisplatin, fluorouracil, radiotherapy, and surgery compared with surgery alone for esophageal cancer: CALGB 9781. J Clin Oncol 26: 1086-92.

12. Kleinberg L, Gibson MK, Forastiere AA (2007) Chemoradiotherapy for localized esophageal cancer: regimen selection and molecular mechanisms of radiosensitization. Nat Clin Pract Oncol 4: 282-94.

13. Rice TW, Blackstone EH, Rybicki LA, Adelstein DJ, Murthy SC, et al. (2003) Refining esophageal cancer staging. J Thorac Cardiovasc Surg 125 : 1103-13.

14. Cooper JS, Guo MD, Herskovic A, Macdonald JS, Martenson JA Jr, et al. (1999) Chemoradiotherapy of locally advanced esophageal cancer: long-term followup of a prospective randomized trial (RTOG 85-01). Radiation Therapy Oncology Group. Jama 281: 1623-7.

15. Shapiro J, van Lanschot JJ, Hulshof MC, van Hagen P, van Berge Henegouwen MI, et al. (2015) Neoadjuvant chemoradiotherapy plus surgery versus surgery alone for oesophageal or junctional cancer (CROSS): long-term results of a randomised controlled trial. The Lancet Oncology 16: 1090-8.

16. Davies AR, Gossage JA, Zylstra J, Mattsson F, Lagergren J, et al. (2014). Tumor stage after neoadjuvant chemotherapy determines survival after surgery for adenocarcinoma of the esophagus and esophagogastric junction. J Clin Oncol 32: 2983-90.

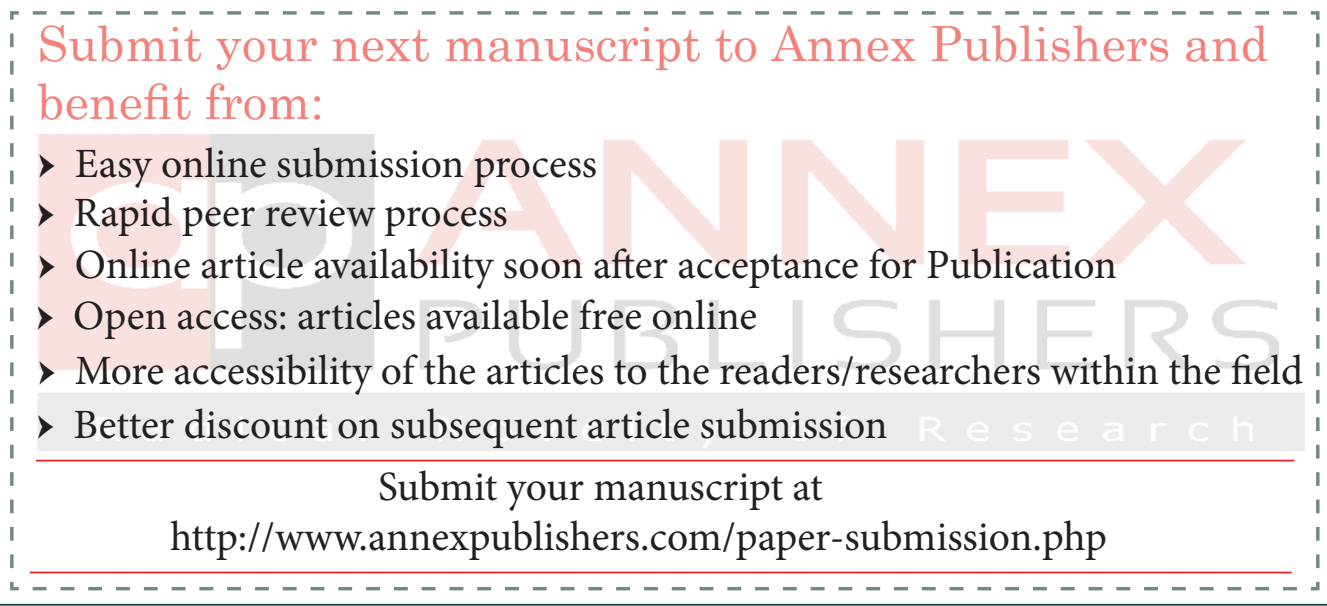

